\title{
Application of the method of auxiliary sources in optical diffraction microscopy
}

\author{
Karamehmedovi, Mirza; Sørensen, Mads Peter; Hansen, Poul-Erik; Lavrinenko, Andrei
}

Published in:

Progress in Industrial Mathematics at ECMI 2008

Link to article, DOI:

10.1007/978-3-642-12110-4_144

Publication date:

2010

Document Version

Early version, also known as pre-print

Link back to DTU Orbit

Citation $(A P A)$ :

Karamehmedovi, M., Sørensen, M. P., Hansen, P-E., \& Lavrinenko, A. (2010). Application of the method of auxiliary sources in optical diffraction microscopy. In Progress in Industrial Mathematics at ECMI 2008 (1 ed., pp. 899-905). Springer. https://doi.org/10.1007/978-3-642-12110-4_144

\section{General rights}

Copyright and moral rights for the publications made accessible in the public portal are retained by the authors and/or other copyright owners and it is a condition of accessing publications that users recognise and abide by the legal requirements associated with these rights.

- Users may download and print one copy of any publication from the public portal for the purpose of private study or research.

- You may not further distribute the material or use it for any profit-making activity or commercial gain

- You may freely distribute the URL identifying the publication in the public portal 


\title{
Application of the Method of Auxiliary Sources in Optical Diffraction Microscopy
}

\author{
M. Karamehmedović ${ }^{1}$, M.-P. Sørensen ${ }^{1}$, P.-E. Hansen ${ }^{2}$, and A. Lavrinenko ${ }^{3}$ \\ 1 DTU Mathematics, Technical University of Denmark, Matematiktorvet 303S, \\ DK-2800 Kgs. Lyngby, Denmark \\ 2 Danish Fundamental Metrology, Technical University of Denmark, \\ Matematiktorvet 307, DK-2800 Kgs. Lyngby, Denmark \\ 3 DTU Fotonik, Technical University of Denmark, Matematiktorvet 303S, \\ DK-2800 Kgs. Lyngby, Denmark
}

\begin{abstract}
Summary. The Method of Auxiliary Sources is used for characterisation of grating defects. Grating profiles are characterised by best fit matching of a library of diffraction efficiencies with numerical simulated diffraction efficiencies with defects. It is shown that the presented method can solve the inverse problem with an accuracy usually thought to require rigorous electromagnetic theories.
\end{abstract}

\section{Characterisation of Micro and Nano Structures Embedded in Materials}

Functional materials with embedded micro and nano structures find application in such diverse areas of technology as optical telecommunication components, self-cleaning windows, medical equipment, and the technology of mass production of electronics and digital watermarks. The main useful properties of such materials are not intrinsic, but rather stem from the introduced modifications on or just beneath the surface of the material. The modifications are, e.g., insertion of particles or air holes of micro or nano scale under the material surface, and alterations of the topology of the surface, such as the introduction of surface gratings or deposition of particles, on micro and nano scale. The design process and industrial use of functional materials require rapid and non-destructive techniques of characterisation of the embedded micro and nano structures. Among several physically distinct methods, we focus on the combined spectroscopic and angular resolved scatterometry technique called Optical Diffraction Microscopy (ODM) [1, 2, 5, 6, 7]. Here, specific features of the sample under investigation are reconstructed from the measured optical power in the scattered far field. The method thus requires the solution of an inverse scattering problem, and ultimately of a nonlinear optimisation problem; however, in an industrial context such as quality con- 
trol, the principal features of the scatterer may be well-known, and one needs rapid interpretation of measurement results to identify only relatively small perturbations, e.g., manufacturing errors, in these features. The structures of interest are typically small in terms of the wavelength of the illuminating light, and it is therefore relevant to address the inverse scattering problem using the full classical electromagnetic model, rather than asymptotic formulations. The Method of Auxiliary Sources (MAS) is an efficient numerical, non-asymptotic technique of solution of boundary problems; see $[4,8]$ and references therein. In the following, the method is used to approximate the solution of example inverse problems which arise in Optical Diffraction Microscopy.

\section{The Method of Auxiliary Sources}

In the context of two-dimensional, time-harmonic forward electromagnetic scattering, the Method of Auxiliary Sources (MAS) is a variational method characterised by the choice of fundamental solutions of the Helmholtz equation in $\mathbb{R}^{2}$ for the expansion vectors of the scattered field, and the Dirac delta functions for the test vectors. Recall that, for every positive $k$, an outgoing ${ }^{4}$ fundamental solution of the Helmholtz equation $\left(\Delta+k^{2}\right) u=0$ in $\mathbb{R}^{2}$, with singularity at $x^{\prime} \in \mathbb{R}^{2}$, is the Hankel function $H_{0}^{(2)}\left(k\left|x-x^{\prime}\right|\right)$ of order zero and of second kind. Figures 1 and 2 show a model time-harmonic Dirichlet scattering problem in $\mathbb{R}^{2}$ and a corresponding MAS formulation. The constant $k$ is the wave number $2 \pi / \lambda$, where $\lambda$ is the operating wavelength. In MAS, all employed fundamental solutions have singularities in the interior of the scatterer. The current sources of the approximation of the exact scattered field - the so-called auxiliary sources - are hence Delta functions in $\mathbb{R}^{2}$ with singularities in the interior of the scatterer, and, in the transverse electric (TE) case, the scattered field $E^{\mathrm{s}}$ is approximated in the exterior $\Omega$ by a finite linear combination of the form $E^{\mathrm{MAS}}(x)=\sum_{j=1}^{N} C_{j} H_{0}^{(2)}\left(k\left|x-x_{j}^{\prime}\right|\right), x \in \Omega$. The weights (complex numbers $C_{j}$ ) occurring in the linear combination are determined by enforcing the boundary condition at selected points $x_{l}, l=1, \ldots, N$, on the scatterer boundary $\Gamma$. The classical inverse scattering problem which arises in the ODM consists in finding a surface $\Gamma$ and a surface current distribution $J$ on $\Gamma$ such that the corresponding radiated far field has the same power pattern as the measured field. Evaluation of the objective function of this nonlinear optimisation problem necessarily involves the evaluation of the intermediate scattered far fields. In this context, the MAS representation of scattered fields holds two major advantages over the traditional surface integrals which originate from boundary layer potential formulations of scattering problems. First, with the MAS formulation, there is no need for numerical integration of surface currents, whereas the electric field radiated by a $z$-directed, time-harmonic electric current distribution $J$ on a boundary $\Gamma$ in $\mathbb{R}^{2}$ is proportional to the

\footnotetext{
${ }^{4}$ That is, satisfying the outgoing radiation condition.
} 


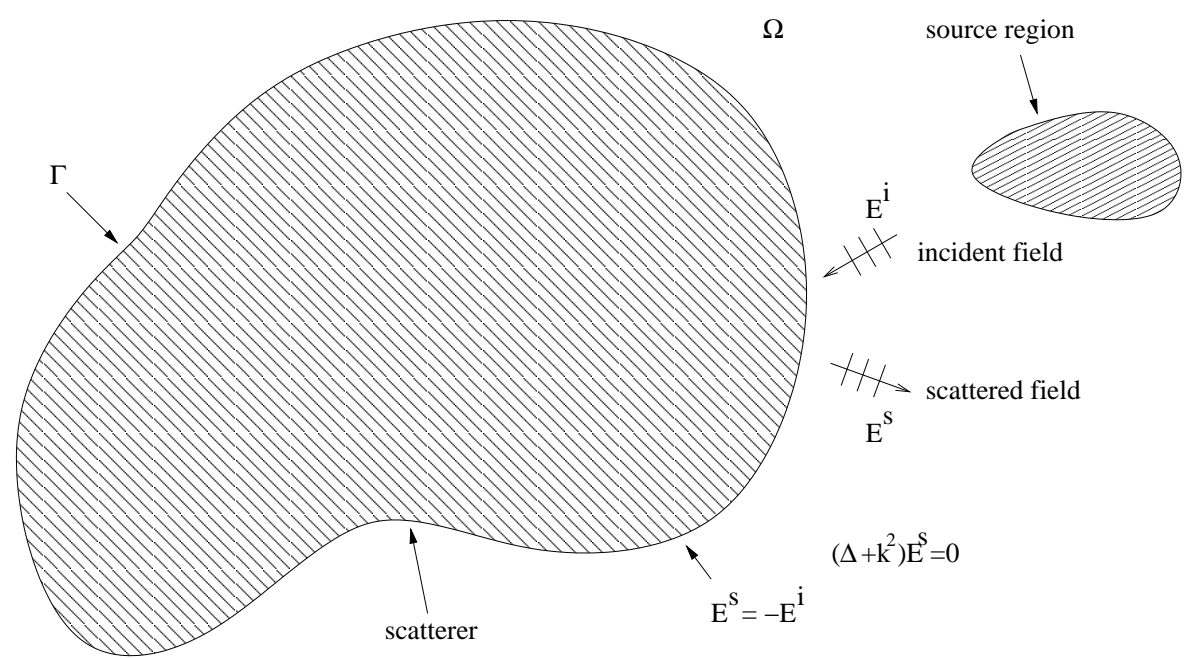

Fig. 1. The geometry of a scattering problem in a subset $\Omega$ of $\mathbb{R}^{2}$.



Fig. 2. A MAS setup used to approximate the solution of the considered boundary problem.

integral $\int_{\Gamma} H_{0}^{(2)}\left(k\left|x-x^{\prime}\right|\right) J\left(x^{\prime}\right) d \Gamma\left(x^{\prime}\right)$, for $x$ in the exterior of $\Gamma$. The second major advantage of the MAS is that the scatterer topology is identified only with the auxiliary sources, rather than with the sources and with a supporting boundary $\Gamma$. In the abovementioned integral, the domain of integration $\Gamma$ is, in general, a parameter of optimisation, and hence needs to be changed with each iteration. In conclusion, when MAS is used, the optimisation problem involves an objective function which is simply a finite sum independent 
of the actual geometry of the scatterer surface, as opposed to an integral taken over a generally variable surface. In our implementation, described in Section 3, a number of scattered far field power patterns are stored in a library, together with the corresponding sets of auxiliary sources. (The latter are represented by the locations $x_{j}^{\prime}, j=1, \ldots, n$, and the complex amplitudes $C_{j}, j=1, \ldots, n$; these sources radiate suitable approximations of the stored far field patterns.) Each far field power pattern corresponds to a well-defined perturbation of the basic topology of the scatterer. With elements $x$ and $x^{\prime}$ of $\mathbb{R}^{2}$ represented by $(|x|, \phi)$ and $\left(\left|x^{\prime}\right|, \phi^{\prime}\right)$, respectively, in the usual cylindrical coordinates, the function $\frac{1+i}{\sqrt{\pi k\left|x-x^{\prime}\right|}} e^{-i k\left|x-x^{\prime}\right|} e^{i k\left|x^{\prime}\right| \cos \left(\phi-\phi^{\prime}\right)}$ is the asymptotic form of the Hankel function $H_{0}^{(2)}\left(k\left|x-x^{\prime}\right|\right)$ of order zero and of second kind, valid for $\left|x-x^{\prime}\right| \gg \lambda$. We use the phase function $e^{i k\left|x^{\prime}\right| \cos \left(\phi-\phi^{\prime}\right)}$ of this asymptotic form for the auxiliary sources in our implementation. The procedure first compares the measured far-field power pattern with the direct samples in the library, using a distance function of the form $\left.\sum|| E^{\text {library }}\left(\phi_{l}\right)|-| E^{\mathrm{m}}\left(\phi_{l}\right)\right|^{2}$, where $\left|E^{\mathrm{m}}\left(\phi_{l}\right)\right|$ is the measured magnitude of the far field at angle $\phi_{l}$. After the best match is found, simple interpolation is used to refine this solution of the inverse scattering problem. The auxiliary sources corresponding to the best match, as well as those corresponding to the two entries in the library which are closest to the best match, are fetched; these sources are represented by complex amplitude vectors $C_{0}, C_{-1}$ and $C_{1}$ in $\mathbb{C}^{N}$, respectively. The objective function, which is a finite sum of the form $\sum|| E_{t}^{\mathrm{MAS}}\left(\phi_{l}\right)|-| E^{\mathrm{m}}\left(\phi_{l}\right)||^{2}$, is then minimised with respect to the parameter $t \in[-1,1]$; the field $E_{t}^{\mathrm{MAS}}$ is radiated by the auxiliary sources represented by the complex amplitude vector $C(t)=-t C_{-1}+(1+t) C_{0}$ when $t \in[-1,0]$, and by $C(t)=(1-t) C_{0}+t C_{1}$ when $t \in[0,1]$. It is here assumed that the library entries are sufficiently close such that the error is, to a good approximation, a linear function of the perturbation of the scatterer geometry. The optimum value of the parameter $t$ is therefore directly interpreted as a normalised deviation of the measured geometry from the library entries.

\section{Results}

Figure 3 shows the two-dimensional scattering problem considered here, and the type of the measured deviations in the scatterer topology. The scatterer, a piece of corrugated silicon, is immersed in air and illuminated by a timeharmonic, uniform plane wave of transverse electric (TE) polarisation and unit amplitude. The incident field propagates in the negative $x$ direction. The operating wavelength is denoted $\lambda$. We want to measure the elongation of a specific protrusion on the scatterer. Our numerical experiment does not use actual field measurements; rather, the amplitude of the scattered electric far field is calculated using the COMSOL software $[3,9]$. The library entries are samples of the magnitude of the scattered electric far field, taken over the angle 


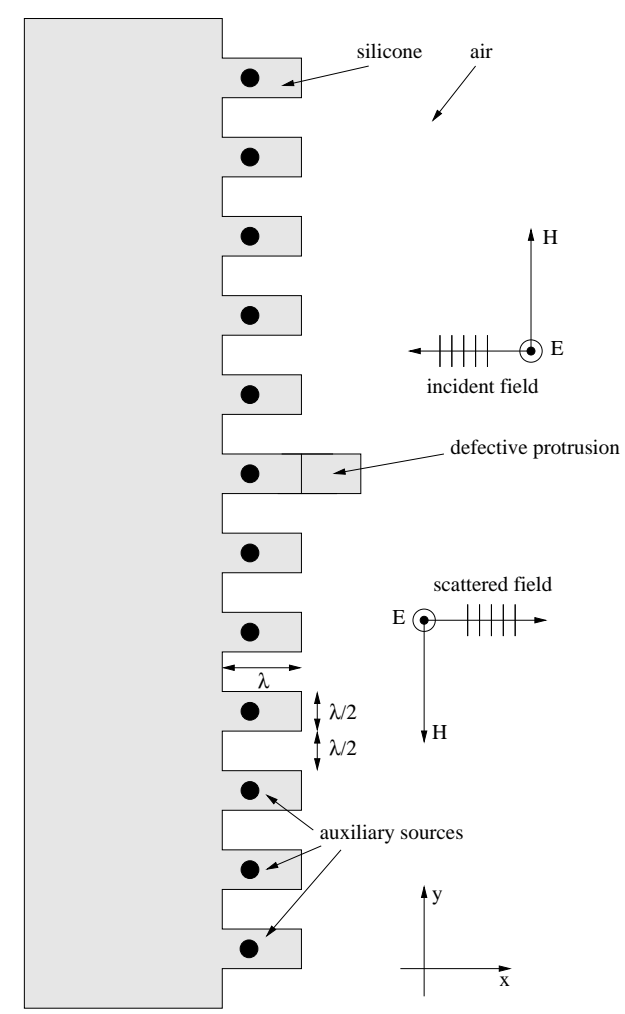

Fig. 3. The type of grating defects to be characterised.

of $30^{\circ}$ symmetrically with respect to the $x$-axis. A total of only 12 auxiliary sources are used for the interpolation of the far fields. Table 1 shows the results of the numerical experiment. The actual and the estimated elongations in the table are shown normalised with respect to the operating wavelength. Negative (positive) elongations correspond to the specific protrusion being shorter (longer) than the nominal one wavelength $\lambda$. Relative error 1 and relative error 2 show the error in the estimate relative to the actual elongation and relative to the nominal protrusion length, respectively. For the results in Table 1, the average absolute value of relative error 1 is $14.7 \%$, and the average absolute value of relative error 2 is $5.4 \%$. Of course, the elongations already represented in the library are measured with zero error, which improves the overall accuracy estimate for the method. However, it also turns out that the elongations of $0.2 \lambda, 0.4 \lambda$ and $0.6 \lambda$ match well the library entries of $0.875 \lambda$ and $1 \lambda$, which suggests that, in general, an appropriate a priori estimate is needed of the possible range of the elongation under measurement. After forcing the correct initial (library) values for the three abovementioned elongations, the interpolation produces estimates with relative error 2 at $12.5 \%,-18.8 \%$ and $-6.0 \%$, respectively. 
Table 1. Accuracy of the estimates of the protrusion elongation.

\begin{tabular}{c|c|c|c} 
actual elongation & estimated elongation & relative error 1 $(\%)$ & relative error 2(\%) \\
\hline-0.9 & -0.9206 & -2.3 & -2.1 \\
\hline-0.3 & -0.1800 & 40.0 & 12.0 \\
\hline-0.2 & -0.1800 & 10.0 & 2.0 \\
\hline-0.1875 & -0.1800 & 4.0 & 0.75 \\
\hline 0.1 & 0.0681 & -31.9 & -3.2 \\
\hline 0.3125 & 0.3250 & 4.0 & 1.3 \\
\hline 0.8125 & 0.6681 & -17.8 & -14.4 \\
\hline 0.9 & 0.9713 & 7.9 & 7.1 \\
\hline
\end{tabular}

\section{Conclusions and Further Work}

It was demonstrated that the Method of Auxiliary Sources can be used for efficient numerical approximation of solution of certain inverse scattering problems occurring in two-dimensional monochromatic Optical Diffraction Microscopy. The method was tested on a number of relevant two-dimensional inverse problems involving the elongation of a specific protrusion in a grating. Future work includes the generalisation of the presented method to threedimensional measurement, and to polychromatic measurement (in time domain).

We acknowledge the financial support from the innovation consortium FINST under the Danish Agency for Science, Technology and Innovation.

\section{References}

1. N. Agersnap, P.-E. Hansen, J. C. Petersen, J. Garnaes, N. Destouches and O. Parriaux, Proc. SPIE Int. Soc. Opt. Eng., 5965, 68-78 (2005)

2. F. Borsetto, K. Carneiro, I. Davi, J. Garnaes, J. C. Petersen, N. Agersnap, P.-E. Hansen, J. Holm and L. H. Christensen, Proc. of the 7th International Conference and 8th General Meeting of the European Society for Precision Engineering and Nanotechnology, Baaden, May 2006

3. COMSOL Multiphysics demonstration CD-ROM can be requested at http://www.comsol.com

4. G. Fairweather and A. Karageorghis, Adv. Comput. Math., 9, 69-95 (1998)

5. J. Garnaes, P.-E. Hansen, N. Agersnap, I. Davi , J. C. Petersen, A. Kuhle, J. Holm and L. H. Christensen, Proc. SPIE Int. Soc. Opt. Eng., 5878, 1-9 (2005)

6. J. Garnaes, P.-E. Hansen, N. Agersnap, J. Holm , F. Borsetto and A. Kuhle, Appl. Opt., 45, 3201-3212 (2006)

7. P.-E. Hansen and L. Nielsen, submitted to Mater. Sci. Eng., B

8. D. I. Kaklamani and H. T. Anastassiu, IEEE Antennas Propag., 44, 48-64 (2002)

9. W. B. J. Zimmerman, Multiphysics Modelling with Finite Element Methods (World Scientific, 2006) 